\title{
Lusioersily
}

\section{Directed neural connectivity changes in robot-assisted gait training: A partial Granger causality analysis}

Youssofzadeh, V., Zanotto, D., Stegall, P., Naeem, M., Wong-Lin, K., Agrawal, S., \& Prasad, G. (2014). Directed neural connectivity changes in robot-assisted gait training: A partial Granger causality analysis. In Unknown Host Publication (pp. 6361-6364). IEEE. https://doi.org/10.1109/EMBC.2014.6945083

Link to publication record in Ulster University Research Portal

\section{Published in:}

Unknown Host Publication

Publication Status:

Published (in print/issue): 01/01/2014

DOI:

10.1109/EMBC.2014.6945083

\section{Document Version}

Author Accepted version

\section{General rights}

Copyright for the publications made accessible via Ulster University's Research Portal is retained by the author(s) and / or other copyright owners and it is a condition of accessing these publications that users recognise and abide by the legal requirements associated with these rights.

\section{Take down policy}

The Research Portal is Ulster University's institutional repository that provides access to Ulster's research outputs. Every effort has been made to ensure that content in the Research Portal does not infringe any person's rights, or applicable UK laws. If you discover content in the Research Portal that you believe breaches copyright or violates any law, please contact pure-support@ulster.ac.uk. 


\title{
Directed neural connectivity changes in robot-assisted gait training: A partial Granger causality analysis
}

\author{
Vahab Youssofzadeh ${ }^{1}$, Damiano Zanotto ${ }^{3}$, Paul Stegall ${ }^{3}$, Muhammad Naeem ${ }^{2}$ and KongFatt Wong- \\ Lin $^{1}$, Sunil K. Agrawal ${ }^{3^{*}}$, Girijesh Prasad ${ }^{1 *}$
}

\begin{abstract}
Now-a-days robotic exoskeletons are often used to help in gait training of stroke patients. However, such robotic systems have so far yielded only mixed results in benefiting the clinical population. Therefore, there is a need to investigate how gait learning and de-learning get characterised in brain signals and thus determine neural substrate to focus attention on, possibly, through an appropriate brain-computer interface (BCI). To this end, this paper reports the analysis of EEG data acquired from six healthy individuals undergoing robot-assisted gait training of a new gait pattern. Time-domain partial Granger causality (PGC) method was applied to estimate directed neural connectivity among relevant brain regions. To validate the results, a power spectral density (PSD) analysis was also performed. Results showed a strong causal interaction between lateral motor cortical areas. A frontoparietal connection was found in all robot-assisted training sessions. Following training, a causal "top-down" cognitive control was evidenced, which may indicate plasticity in the connectivity in the respective brain regions.
\end{abstract}

\section{INTRODUCTION}

An important challenge in the field of neuroscience and brain-computer interface $(\mathrm{BCI})$ is to characterise causal connectivity between brain regions during a specific task. Granger causality (GC) is a useful data-driven approach to search for the causal influence of one time-series on another [1]. The GC application to EEG studies is controversial, mainly due to unknown confounding effects (e.g., exogenous inputs and latent variables), and to the stationarity assumption of the underlying stochastic process in multivariate autoregressive (MVAR) analysis and statistical bias [2].

Time-domain partial-GC (PGC) analysis specifically mitigates confounding influences caused by endogenous latent variables and exogenous environmental inputs [3]. It modifies the standard GC measure by adding terms based on residual correlations between the predicted and the conditional variables. It assumes that all confounding influences are reflected in correlations with the residuals and

V.Y., G.P. and K.W.-L. are with Intelligent Systems Research Centre (ISRC), Faculty of Computing and Engineering, University of Ulster, Magee Campus, Northland Road, BT48 7JL, Northern Ireland (phone: +44 28 71675645; e-mail: Prasad, Girijesh <g.prasad@ulster.ac.uk>).

D.Z., P.S. and S.K.A. are with Rehabilitation and Robotics (ROAR) Lab, Columbia University, New York, NY, 10027 USA.

N.M is with Institute of Digital Health, University of Warwick, Coventry, UK. therefore can be detected and partly be factored out, similar to partial correlation [3]. To the best of our knowledge, the application of this useful tool to BCI type datasets and, more specifically, to robot-assisted gait training has not been tested before. In a number of studies, our group has shown that training with Active-Leg Exoskeleton (ALEX) can result in short-term adaptations in the gait of healthy subjects and those with strokes [4], [5].

Following our previous work in [6], we apply a timedomain PGC to the EEG data acquired during a robotassisted gait training paradigm to investigate the functional directed connectivity of cortical networks. In our investigations, we use five scalp EEG electrodes, which include frontal $(\mathrm{Fz})$, central $(\mathrm{Cz})$, left central $(\mathrm{C} 3)$, right central $(\mathrm{C} 4)$ and centro-parietal $(\mathrm{CPz})$ areas. To address the nonstationarity and bias issues, we use EEG short segments and apply permutation analysis. Moreover, the power spectral densities (PSDs) of the EEG segments by means of fast Fourier transform (FFT) are analysed. Finally, we look for consistencies between PSD and PGC results. The interpretations about each method is further discussed in Section III. Our results suggest that PGC measure can play a crucial role in the BCI-gait studies as a model-free and reliable method for deciphering the temporal changes in the functional cortical interactions.

\section{MATERIAL AND METHOD}

\section{A. EEG Data}

The EEG data were collected using g.tec's g.USBamp recording system with 16 active scalp electrodes, while six healthy male adults (age $26.5 \pm 6.5$ years, weight $77.8 \pm 9.7 \mathrm{~kg}$, height $1.79 \pm 0.04 \mathrm{~m}$ ) performed gait training on the ALEX II (Active Leg Exoskeleton, [7]). Each experiment involved a single visit to our laboratory. The robotic leg was attached to the subject's non-dominant leg (i.e., the left leg for all subjects), and the treadmill speed was set to the subject's comfortable walking speed $(0.87 \pm 0.15 \mathrm{~m} / \mathrm{s})$. The subjects underwent robot-assisted gait training, consisting of ten sequential trials: (1) a 10 min bout of free treadmill walking without the robot (FW1); (2) a 5-min baseline test (BSL), where the robot was controlled in transparent mode (i.e., nil interaction with the user); (3-6) four 10-min training bouts (TR), during which the subjects were asked to match an altered footpath derived from their baseline one; (7-9) three 5-min bouts of post-training trials (PT), where the robot was controlled in transparent mode; (10) a 5-min bout of free treadmill walking (FW2). Breaks (2-4 min) were included in- 
between subsequent trials (Fig. 1). During each training session, subjects received haptic guidance (frequency 100\%) and visual guidance (frequency 50\%) from the ALEX II. The former involved assistive forces exerted by the robot to guide the user's leg along the target footpath; the latter consisted of a computer screen located in front of the user, showing the target footpath along with the current foot position. More details about this training protocol can be found in [4]. Prior to each trial, baseline EEG (so called resting stage) activities were recorded for one minute, as the subject remained standstill on the treadmill. We considered this part of the data as a benchmark for stability check.

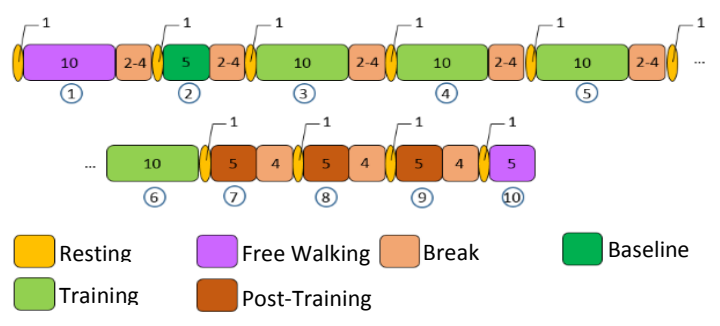

Fig. 1. EEG gait recording protocol.

\section{B. Partial Granger Causality (PGC)}

Based on GC rules, if the prediction error of $X_{t+1}$ using $p$ previous values of $X_{t}$ and $Y_{t}$ (full model) is less than the prediction error using only the series $X_{t}$ (restricted model), then $Y_{t}$ is said to have a causal influence (i.e. Grangercausality) on $X_{t}\left(\mathcal{F}_{Y \rightarrow X}\right)$. Similarly, if the prediction error of $Y_{t}$ in the full model is less than that in the restricted, then $X_{t}$ is said to Granger-cause $Y_{t}\left(\mathcal{F}_{X \rightarrow Y}\right)[1]-[3]$.

$$
\begin{aligned}
& \left\{\begin{array}{l}
X_{t}=\sum_{k=1}^{p} a_{1 i} X_{t-k}+\sum_{i=1}^{p} c_{1 i} Z_{t-k}+\overbrace{\varepsilon_{1 t}+\varepsilon_{1 t}^{E}+B_{1}(L) \varepsilon_{1 t}^{L}}^{U_{1 t}} \\
Y_{t}=\sum_{k=1}^{p} b_{1 i} Y_{t-k}+\sum_{i=1}^{p} d_{1 i} Z_{t-k}+\overbrace{\varepsilon_{2 t}+\varepsilon_{2 t}^{E}+B_{2}(L) \varepsilon_{2 t}^{L}}^{U_{2 t}}
\end{array}\right. \\
& \left\{\begin{array}{c}
X_{t}=\sum_{k=1}^{p} a_{2 i} X_{t-k}+\sum_{i=1}^{p} b_{2 i} Y_{t-k}+\sum_{i=1}^{p} c_{2 i} Z_{t-k} \\
+\overbrace{\varepsilon_{3 t}+\varepsilon_{3 t}^{E}+B_{3}(L) \varepsilon_{3 t}^{L}}^{U_{3 t}} \\
Y_{t}=\sum_{k=1}^{p} d_{2 i} X_{t-k}+\sum_{i=1}^{p} e_{2 i} Y_{t-k}+\sum_{i=1}^{p} f_{2 i} Z_{t-k} \\
+\overbrace{\varepsilon_{4 t}+\varepsilon_{4 t}^{E}+B_{4}(L) \varepsilon_{4 t}^{L}}^{U_{4 t}}
\end{array}\right. \\
& S=\left[\begin{array}{cc}
\operatorname{var}\left(u_{1 t}\right) & \operatorname{cov}\left(u_{1 t}, u_{2 t}\right) \\
\operatorname{cov}\left(u_{2 t}, u_{1 t}\right) & \operatorname{var}\left(u_{2 t}\right)
\end{array}\right] \\
& \Sigma=\left[\begin{array}{cc}
\operatorname{var}\left(u_{3 t}\right) & \operatorname{cov}\left(u_{3 t}, u_{4 t}\right) \\
\operatorname{cov}\left(u_{4 t}, u_{3 t}\right) & \operatorname{var}\left(u_{4 t}\right)
\end{array}\right] \\
& \left\{\begin{array}{l}
\mathcal{F}_{Y \rightarrow X \mid Z}=\operatorname{Ln}\left(\frac{S_{11}-S_{12} S_{22}^{-1} S_{21}}{\sum_{11}-\Sigma_{12} \Sigma_{22}^{-1} \Sigma_{21}}\right) \\
\mathcal{F}_{X \rightarrow Y \mid Z}=\operatorname{Ln}\left(\frac{S_{22}-S_{21} S_{11}^{-1} S_{12}}{\Sigma_{22}-\Sigma_{21} \Sigma_{11}^{-1} \Sigma_{12}}\right)
\end{array}\right.
\end{aligned}
$$

In PGC, it is assumed that two typical independent sources, $X_{t}$ and $Y_{t}$ are influenced by latent variables
( $L_{1}$ and $\left.L_{2}\right)$, exogenous inputs $\left(E_{1}\right.$ and $\left.E_{2}\right)$ and the modulatory factor (driven by series $Z_{t}$ ). The corresponding residual error of two confounding factors $\varepsilon_{t}^{E}$ ( $E$ : exogenous) and $B(L) \varepsilon_{t}^{L} \quad\left(L\right.$ : latent) along with residual error $\varepsilon_{t}$ can be incorporated into MVAR representation of the restricted model (Eq. 1) and full model (Eq. 2). $B(L)$ is the polynomial matrix of appropriate lag operator $L$ related to latent input, which depends on its history. Considering the covariance term to control the indirect interactions (Eq. 3), the causal influence by PGC (e.g., $\mathcal{F}_{Y \rightarrow X \mid Z}$ ) is computed by the log-ratio of the corresponding residual errors (Eq. 4). Finally, to statistically extract the significant causal effects, the logarithmic term can be tested using surrogate data techniques (e.g. permutation or bootstrapping) [8].

\section{Data Analysis}

We selected five electrodes covering the most important brain areas involved during locomotion, including pre-frontal $(\mathrm{Fz})$, motor $(\mathrm{C} 3, \mathrm{Cz}, \mathrm{C} 4)$ and centro-parietal $(\mathrm{CPz})$ areas. The data was pre-processed using a band-pass filter in the range 0.5-30 Hz to purge unwanted ultra-low (close to DC) and high frequency noises. Although the gamma brain activities $(25-40 \mathrm{~Hz})$ are suppressed, this frequency range is considered as usual band width analysed in BCI or motor imagery control. The trials in which the signal on any channels exceeded $80 \mu \mathrm{V}$ were marked as artefacts and rejected. Hence, an average $10 \%$ of trials were rejected from responses of each subject. In order to eliminate inevitable movement artefacts (eye movements and eye blinks, muscle activities, treadmill vibrations), we applied infomax ICA [9]. Along with visual inspection, the average of first four ICs was rejected. The reconstructed data were then baseline corrected. To prevent confounds due to nonstationarity, we divided the data into approximately 5 seconds (i.e., 2560 time samples), stationary, non-overlapping short windows and applied a linear detrending process to each segment. The segmentation, thus, yielded 120 segments during each training trial (TR) and 60 during each post-training trial (PT). We computed for each segment the optimal model order $p$ (i.e., the number of time-lags) in the range of $1 \leq p \leq 20$ using Akaike information criterion (AIC). To confirm the validity of application of regression models we used the consistency check of the correlation structure as in [10]. It provides a value close to 1 if the correlation of simulated data is close to the correlation of evaluating data. Finally, to systematically remove the statistical bias when performing significance tests, the permutation-resampling test was used. We selected the number of permutations of $n=20$ and the window size of $L=20$ samples. The computations related to data pre-processing and extracting causal effects were performed using EEGLab (V9.0.4b) [11] and GCCA (V2.9) toolbox [8], respectively.

\section{RESULTS}

The PSD and PGC analyses were conducted on artefactfree EEG segments. Fig. 2 shows the average results of both PSD and PGC analyses at group level. Both PSD and PGC results were separately normalised in $U(0,1)$. Each analysis 
was divided into 10 blocks of resting (i.e., quiet standing prior to each trial) and task activities (i.e., the ten walking

bouts).

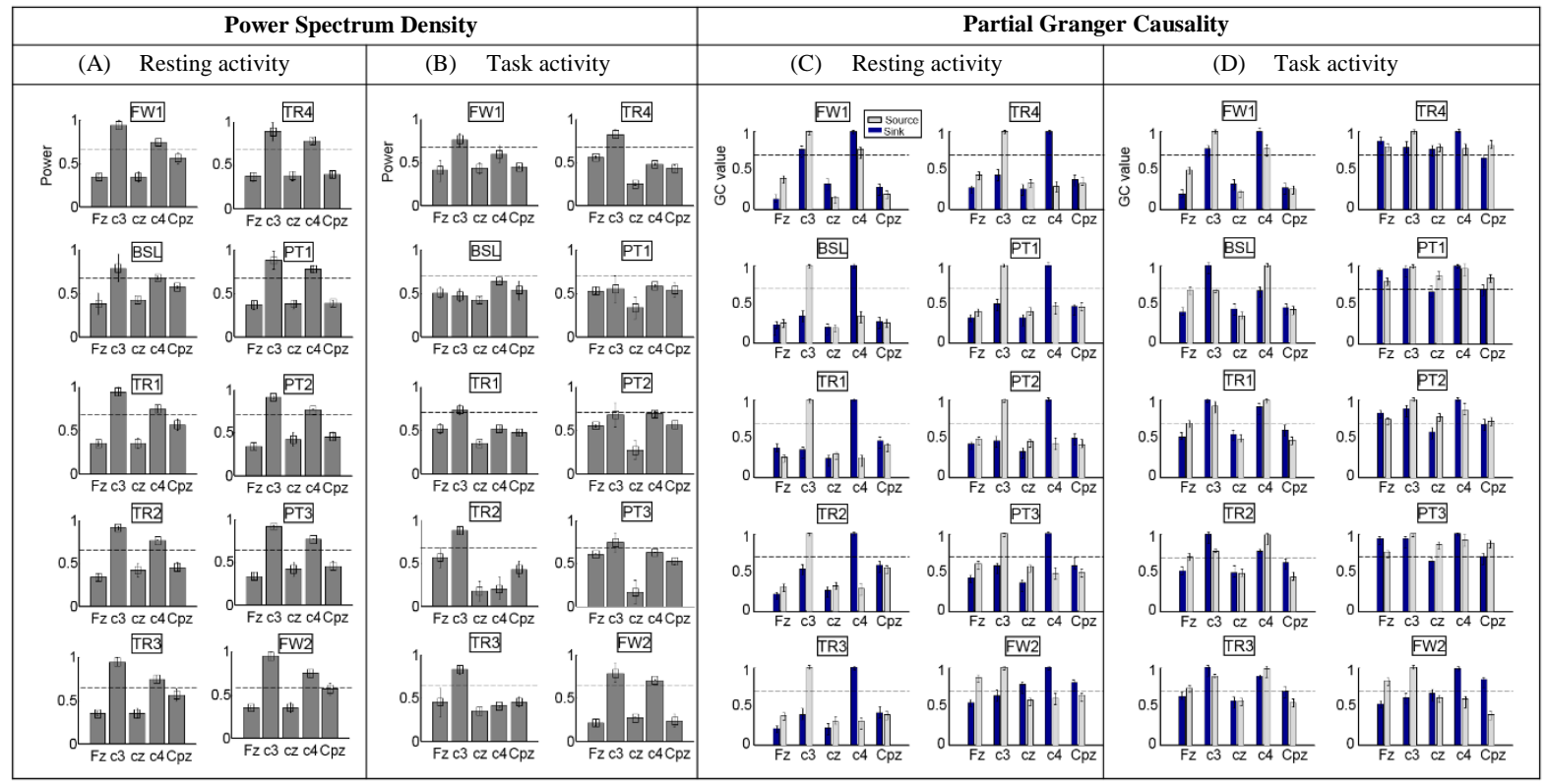

Fig. 2. Power spectrum density (PSD) and partial Granger causality analysis (PGC) of EEG-gait training data in 10 sequential trial runs. The trials include, (1) free walk (FW1), (2) gait within the exoskeleton for baseline (BSL), (3-6) gait training with 3 modified templates (TR1-4), (7-9) gait post-training without templates (PT1-3), and (10) gait post-training in free walk (FW2). Error bars represent standard errors. Mean and standard error are computed across 5 second windows of data.

The average empirical model order and the average consistency test values were $p=10$ and $C=91 \%$. This satisfactory value of the consistency test proves the capability of the model to reproduce the EEG time series. PGC values greater than a threshold of $0.7(p$-value $=0.1)$ were assumed to indicate a significant causal effect. The avarge of estimated outgoing and incoming causal effects inferred by PGC are labelled as "source" and "sink", respectively. In the resting stage (Fig. 2A and 2C), similar patterns were observed in PSD and PGC results, which confirm consistency of the PGC method. For instance, in Fig. 2A, PSD shows significant activities in the lateral motor cortical areas (i.e. power in $\mathrm{C} 3$ is greater than that in $\mathrm{C} 4$ ). Similarly, PGC disclosed a significant outgoing causal influence at $\mathrm{C} 3$ and significant incoming causal influence at $\mathrm{C} 4$. To highlight the differences between resting and task performances, we investigated the ensemble average of PSDs (average across all EEG channels and for all subjects) as presented in Fig.3. Interestingly, in resting stage the measurements showed an increased activity in alpha band (8$13 \mathrm{~Hz}$ ) while the task measurements revealed a major peak at theta $(4-7 \mathrm{~Hz})$ band. Moreover, in the PSD results of FW1 and FW2 (first and last free walk trials), the resting and task performance gave rise to almost similar results (albeit a slight theta peak in FW2), indicating consistency of the technique. Next, we translated the network causal effects of task activities into a scalp map representation (i.e. without averaging the GC values across channels) as shown in Fig. 4. It can be seen that the pattern of causal effect is almost similar in FW1 and FW2 (except for the effect of $\mathrm{Fz} \rightarrow \mathrm{Cz}$ ). Moreover, a strong casual interaction was found between $\mathrm{C} 3$ and $\mathrm{C} 4$ in almost all states, a motor area frequently reported in BCI studies [12]. It is noteworthy that the effect of $\mathrm{C} 3 \rightarrow \mathrm{C} 4$ was stronger than $\mathrm{C} 4 \rightarrow \mathrm{C} 3$ in all cases - the asymmetry being sharper in the trials not involving the robot. This can be also inferred by inspecting the average results in Fig. 2C and 2D. Previous studies have documented asymmetries in the medial regions of the primary sensory motor cortex during treadmill walking, and associated them with foot-dominance [13]. This hypothesis is also applicable to our results, given that all subjects were right-leg dominant. We further speculate that the reduced level of asymmetry measured in all the sessions involving the robot was induced by ALEX II, the device being unilateral (i.e., the robotic leg was attached to the subject's left leg, while the right leg was free).
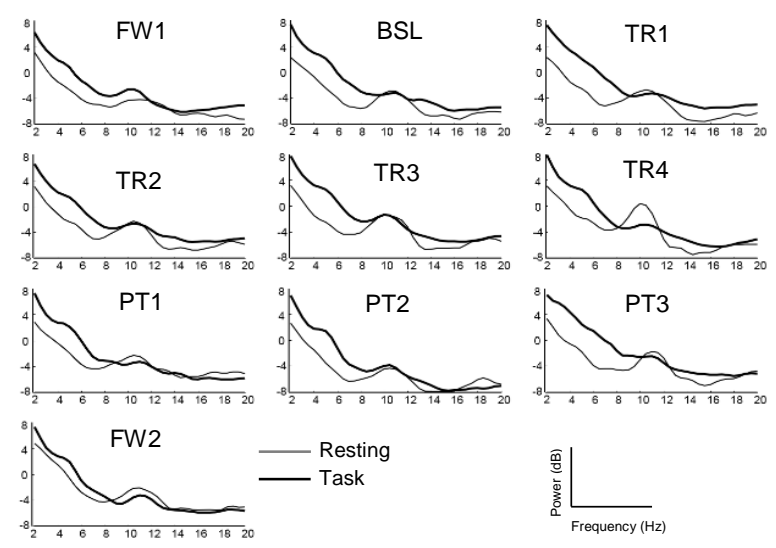

Fig.3. Mean (log) power spectral density of all EEG channels in all 10 blocks. The results consist of resting and task performances. 
All the training sessions evidenced a fronto-parietal connection $(\mathrm{Fz} \rightarrow \mathrm{CPz})$ : previous studies have demonstrated the existence of such network in tasks involving rapid visual information processing [14]. Indeed, the visual feedback provided during training required sustained attention to the mismatch between the prescribed footpath and the current foot position (frontal cortex), along with visuospatial processing to convert the abstract representation of the foot motion - displayed in front of the subject - to useful spatial information (parietal lobe). Comparisons between training and post-training trials evidenced a causal "top-down" cognitive control effect $(\mathrm{Fz} \rightarrow \mathrm{Cz})$ during post-training trials. While the medial portion of the primary motor cortex is actively involved in normal walking, involvement of the prefrontal cortex in locomotor control has been related to motor adaptation of the gait pattern in healthy subjects [15], and recovery of locomotor function in stroke patients [16]. This interaction changes from unidirectional to bidirectional at trial 9 (PT3), perhaps suggesting a form of plasticity in the connectivity between these brain regions [17].

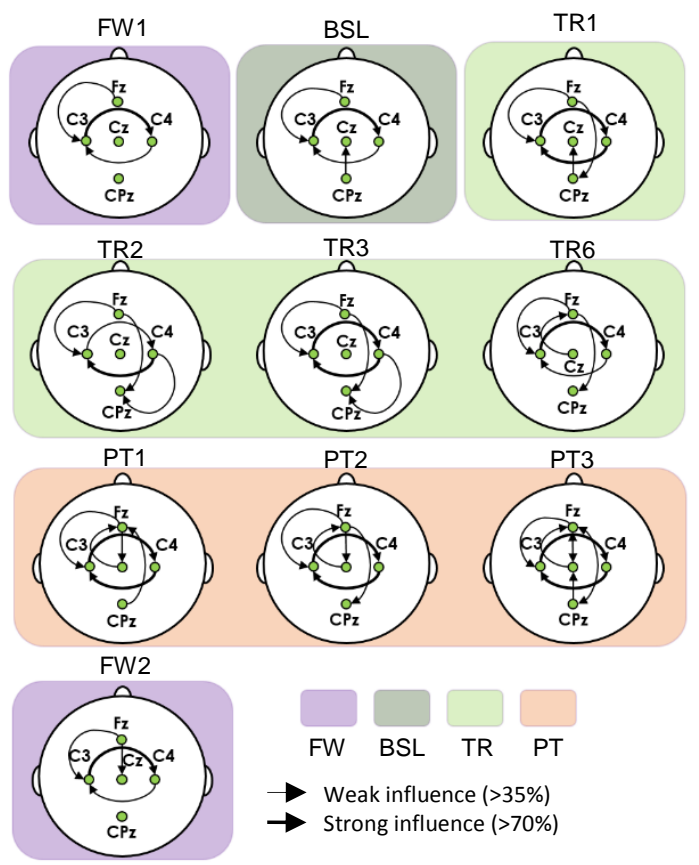

Fig. 4. Mean partial Granger causality (PGC) of EEG segments in all 10 blocks.

\section{CONCLUSION}

In this work, we applied the time-domain partial Granger causality method (PGC) to study directed neural connectivity in robot-assisted training of modified gait patterns. The validity of the method was assessed by comparing PSD and PGC data. Preliminary results evidenced changes in neural connectivity pre, during and post training trial runs. Future work will include correlating such changes to subjects' motor adaptations, including gait kinematics, gait timing and muscle activity.

\section{REFERENCES}

[1] C. W. J. Granger, "Investigating Causal Relations by Econometric Models and Cross-spectral Methods," Econometrica, vol. 37, pp. 424-438, 1969.

[2] A. B. Barrett, M. Murphy, M.-A. Bruno, Q. Noirhomme, M. Boly, S. Laureys, and A. K. Seth, "Granger causality analysis of steady-state electroencephalographic signals during propofol-induced anaesthesia," PLoS One, 7(1), 2012.

[3] S. Guo, A. K. Seth, K. M. Kendrick, C. Zhou, and J. Feng, "Partial Granger causality--eliminating exogenous inputs and latent variables.," J. Neurosci. Methods, 172(1), Jul. 2008.

[4] D. Zanotto, G. Rosati, S. Spagnol, P. Stegall, and S. Agrawal, "Effects of complementary auditory feedback in robotassisted lower extremity motor adaptation," IEEE Trans. Neural Syst. Rehabil. Eng., 21(5), pp. 775-86, 2013.

[5] L. E. Alex, S. K. Banala, S. H. Kim, S. Agrawal, J. P. Scholz, "Robot Assisted Gait Training With Active," 17(1), 2009.

[6] V. Youssofzadeh, G. Prasad, M. Naeem, and K. Wong-Lin, "Partial Granger Causality Analysis for Brain Connectivity based on Event Related Potentials," in Front. Neuroinform. Conference Abstract: Neuroinformatics 2013, 2013, no. 114.

[7] P. Stegall, K. Winfree, D. Zanotto, and S. Agrawal, "Rehabilitation Exoskeleton Design: Exploring the Effect of the Anterior Lunge Degree of Freedom,", 29(4), 2013.

[8] A. K. Seth, "A MATLAB toolbox for Granger causal connectivity analysis.," J. Neurosci. Methods, vol. 186, no. 2, pp. 262-73, Feb. 2010.

[9] A. J. Bell and T. J. Sejnowski, "An information-maximization approach to blind separation and blind deconvolution.," Neural Comput., vol. 7, pp. 1129-1159, 1995.

[10] M. Ding, S. L. Bressler, W. Yang, and H. Liang, "Shortwindow spectral analysis of cortical event-related potentials by adaptive multivariate autoregressive modeling: data preprocessing, model validation, and variability assessment.," Biol. Cybern., vol. 83, no. 1, pp. 35-45, Jul. 2000.

[11] A. Delorme and S. Makeig, "EEGLAB: an open source toolbox for analysis of single-trial EEG dynamics including independent component analysis.," J. Neurosci. Methods, vol. 134, no. 1, pp. 9-21, Mar. 2004.

[12] B. Várkuti, C. Guan, Y. Pan, K. S. Phua, K. K. Ang, C. W. K. Kuah, K. Chua, B. T. Ang, N. Birbaumer, and R. Sitaram, "Resting state changes in functional connectivity correlate with movement recovery for BCI and robot-assisted upperextremity training after stroke.," Neurorehabil. Neural Repair, vol. 27, no. 1, pp. 53-62, Jan. 2013.

[13] I. Miyai, H. C. Tanabe, I. Sase, H. Eda, I. Oda, I. Konishi, Y. Tsunazawa, T. Suzuki, T. Yanagida, and K. Kubota, "Cortical mapping of gait in humans: a near-infrared spectroscopic topography study.," Neuroimage, 14, pp. 1186-1192, 2001.

L. Wang, X. Liu, K. G. Guise, R. T. Knight, J. Ghajar, and J. Fan, "Effective connectivity of the fronto-parietal network during attentional control.," J. Cogn. Neurosci., 22, 2010.

M. Suzuki, I. Miyai, T. Ono, I. Oda, I. Konishi, T. Kochiyama, and K. Kubota, "Prefrontal and premotor cortices are involved in adapting walking and running speed on the treadmill: An optical imaging study," Neuroimage, vol. 23, pp. 1020-1026, 2004.

[16] M. Mihara, I. Miyai, M. Hatakenaka, K. Kubota, and S Sakoda, "Sustained prefrontal activation during ataxic gait: A compensatory mechanism for ataxic stroke?," Neuroimage, vol. 37, pp. 1338-1345, 2007.

[17] I. Momennejad and J. D. Haynes, "Human anterior prefrontal cortex encodes the 'what' and 'when' of future intentions," Neuroimage, vol. 61, pp. 139-148, 2012. 